\title{
Audit of the Management of Primary Hyperparathyroidism at Watford General Hospital
}

Matthew Allum, Michael R Clements

\section{Introduction}

Primary hyperparathyroidism (PHPT) can only be cured by parathyroidectomy (PTX) but mild and asymptomatic cases can often be managed conservatively. We carried out a retrospective audit of patients attending the Endocrine Clinic at Watford to determine how our practice compared with the different guidelines for surgical referral published in the US and the UK.

\section{Method}

A search was carried out of clinic letters from Jan 2010 to Oct

2011. Additional data were gathered from the hospital

biochemistry results system. We determined the number of patients who fulfilled either of two sets of criteria for surgery and whether or not they had yet been referred for PTX.

\section{Criteria}

UK Criteria, including symptomatic PHPT (Davies 2002) ${ }^{1}$

- Serum Calcium > $3.0 \mathrm{mmol} / \mathrm{L}$

- Renal calculi

- Acute pancreatitis

- Age $<50$ years

- Osteoporosis at hip or spine

- 24 urine calcium excretion $>10 \mathrm{mmol} /$ day

US surgical criteria for asymptomatic PHPT (NIH 2008) ${ }^{2}$

- Serum calcium > 2.8mmol/L (0.25 above upper limit of normal)

- GFR $<60 \mathrm{ml} / \mathrm{min}$

- Osteoporosis $(T<-2.5)$

- Age $<50$ years

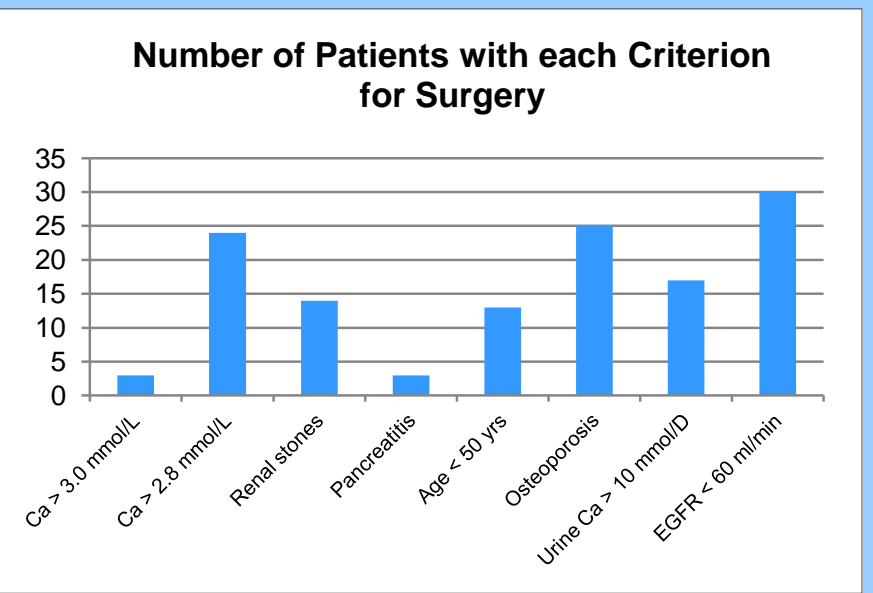

West Hertfordshire Hospitals NHS Trust

Results
97 patients were identified with PHPT. 32 of these patients
were referred for PTX. 55 patients had indications for
surgery according to UK guidelines and 29 of these (53\%)
had so far been referred. Of those not referred the
indication for surgery in 16 was osteoporosis only. 71
patients fulfilled US criteria for PTX and 31 of them ( $44 \%$ )
had been referred. 12 patients not yet submitted to PTX had
a GFR < $60 \mathrm{ml} / \mathrm{min}$ as the only indication for surgery. Mean
age of patients not yet referred was 72.6 yrs (median 74
yrs).

\section{Number of Patients with PHPT Referred for Surgery, by Criteria Set}

- Total Of Which Referred for Surgery

97

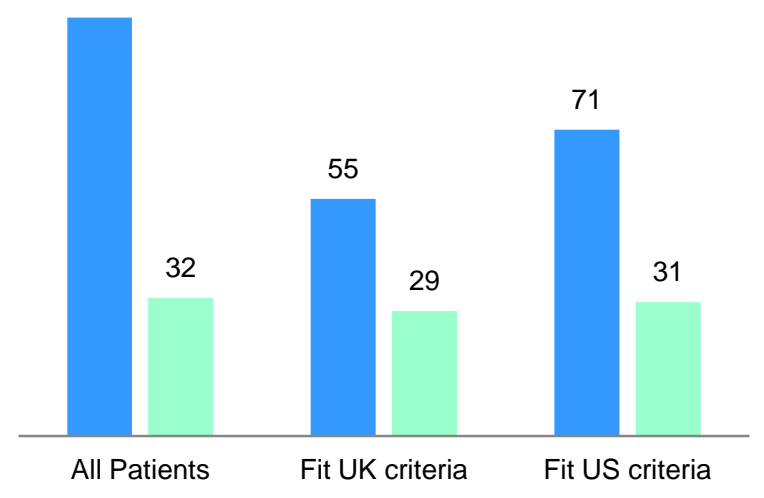

\section{Conclusion}

Our practice in this cohort was relatively conservative. Approximately half of patients who fulfilled some criterion for surgery (US or UK) had not been referred. Reasons for this included age, stable disease, co-morbidities and patient choice. Further explanation from retrospective analysis will be available and a prospective audit is also underway.

\section{References}

1 Davies M, Fraser WD, Hosking DJ. The Management of Primary Hyperparathyroidism. Clin Endocrinol (Oxf). 2002 Aug; 57(2):145-55

2 Guidelines for the Management of Asymptomatic Primary Hyperparathryoidism: Summary Statement from the Third International Workshop. John P. Bilezikian, Aliya A. Khan, John T. Potts, on behalf of the Third International Workshop on the Management of Asymptomatic Primary Hyperthyroidism J Clin Endocrinol Metab. 2009 February; 94(2): 335-339 